\title{
Design of P2Pnet: An Autonomous P2P Ad-Hoc Group Communication System
}

\author{
Yao-Nan Lien, Hung-Chin Jang, and Tzu-Chieh Tsai \\ Department of Computer Science, National Chengchi University \\ Taipei, Taiwan, R.O.C. \\ \{lien,jang,ttsai\}@cs.nccu.edu.tw
}

\begin{abstract}
This paper proposes a MANET/VANET based group communication system, P2Pnet, to support the communication and information demand for mobile group users such as rescue teams in catastrophic disasters, army in battle fields, and mobile learning groups. We use the Jiji Earthquake as an example to illustrate the physical environment and to establish system requirements for a mobile group communication system that are suitable for the environments mentioned above. We have implemented the first phase prototype as well as two application experiments on top of P2Pnet: Rescue Information System for Earthquake Disasters (RISED) and a mobile English learning experiment conducted in the Nation Chengchi University.
\end{abstract}

Keyword: Mobile Computing, MANET, P2P Computing.

\section{INTRODUCTION}

As the entire world is benefited from the advance of communication network technologies, mobile users are still limited by physical obstacles. For instance, most users in catastrophic disasters usually lost their communications making rescue and relief operation extremely difficult.

This paper proposes a MANET/VANET based group communication system, P2Pnet, to support the communication and information demand for mobile group users such as rescue teams in catastrophic disasters, army in battle fields, and mobile learning groups. We use the Jiji Earthquake as an example to illustrate the physical environment and to establish system requirements for a mobile group communication system that are suitable for the environments mentioned above.

Almost every year, the world is stricken by numerous catastrophic natural disasters, such as earthquake, hurricane, typhoon, tsunami, etc. When stricken by a catastrophic natural disaster, such as Jiji/Taiwan Earthquake [5], SiChuan/China Earthquake [7], or Hurricane Katrina [6], emergency rescue operation is very critical to numerous lives. Many people trapped in the disastrous areas under collapsed buildings or landslides may have a large chance to survive if they are rescued in 72 hours, referred as "Golden 72 Hours". People evacuated from their home jammed in highways or dome shelters need to communicate to each other for various reasons such as allocation of rescue and relief resource as well as reunion of family members. However, communication systems, fixed or mobile, were usually down due to various reasons. Rescue teams in each stricken area consist of few trained professional squads, army, police, fire fighters, and hundreds of thousands of disorganized volunteers. The loss of communication systems made the rescue operation extremely difficult. In Jiji Earthquake, it took ChungHwa Telecom, the largest telecommunication operator in Taiwan, 15 days of $24 / 7$ operation to restore its mobile communication systems. Many people died before they got a chance to be rescued.

Although establishing a temporary communication network to support emergency communications and networking is one of the most urgent tasks in a disastrous rescue mission, feasible technology options are very limited in such a catastrophic disaster. We propose to use WiFi-ready notebook PCs owned by rescue volunteers themselves to construct a MANET to support such a need. Because the popularity of WiFi-ready notebook PC is very high nowadays, this solution would be highly feasible in many countries.

The platform is designed and implemented in two phases. In the first phase, a simple MANET is implemented to support emergency information system. In the future second phase, we will implement an "Autonomous P2P Ad-Hoc Group Communication Systems (P2Pnet)", which is a local wireless intranet based on P2P and MANET technologies. P2Pnet is used to support the communication need under temporary serverless infrastructure-less Internet-blocked environments such as nature disastrous area, battle-field and mobile learning environments.

Rescue people, voluntary or mission-specific professional, could use their own notebook PCs to construct a multi-hop ad-hoc network to form a basic wireless intranet first, then use our P2Pnet technology to form a higher level missionspecific network to support urgent communication needs such as VoIP, Push-to-Talk, Instant Messaging, and mobile social network, etc.

Due to page limit, this paper will mainly focus on the system analysis and the first phase platform design.

\section{SYSTEM ANALYSIS}

\subsection{Impact and Causes of Communication System Crash}


As mentioned in Section 1, the impact of communication system crash to the Jiji Earthquake was catastrophic. The loss of communication systems and information system created a big impact to the efficiency of rescue operation. A few lessons we learned from the Jiji Earthquake are as follows:

- In a catastrophic disaster, regular rescue teams were far from sufficient for the emergency rescue mission. A large number of volunteers must be mobilized to participate in the rescue operation. However, without a good communication system, it is very difficult to organize and coordinate rescue volunteers.

- Transportation system was paralyzed not only by broken bridges and roads, but also by a large number of disorganized voluntary rescue vehicles.

- A large volume of rescue and relief resources were misplaced because the assessment of disasters distribution is virtually blind and inaccurate in the early hours even days after a big quake. Unfortunately, reallocation of resources may not be possible because of paralyzed transportation systems.

On the other hand, cellular mobile communication systems that were thought highly countable in emergency were completely wiped out in most of the stricken areas. The most common causes are (1) base stations crashed; (2) trunks connecting base stations to MSCs broken; (3) communication systems overwhelmed by extremely huge traffic.

Above mentioned are only parts of the entire list. A more detailed report can be found in [4]. Threatened by so many sources of failure, it required a miracle for a cellular communication system to survive in such a catastrophic disaster, even for a robust system with $99.999 \%$ reliability.

\subsection{Environmental Constraints and System Requirements}

Followings are environmental constraints and requirements.

\section{Environmental Constraints}

- Outgoing link (Internet) is either not available or very limited.

- $\quad$ All Internet based services, such as Skype, are not available, because of no Internet access.

- WiFi-ready notebook PCs and portable power generators are assumed very popular.

- Highways may be jammed by vehicles and many vehicles are VANET (Vehicular ad-hoc Network) ready.

\section{Functional Requirements}

- User interface must be simple, easy to learn, and fool-proof. There is a very stringent time constraint that volunteers won't be able to learn a complicate user interface.

- Devices do not need complicate setup procedure.

- Devices must be fault-tolerant such that misusage will not crash a device.

- The system must support broadcast-based multimedia communications, while unicast communication mode is optional.

- Only basic functions are required, advanced features are optional.

- $\quad$ The system must not demand high power and be rechargeable using a portable power generator.

Without plenty of resources and time, it is not easy to develop a system that meets all the requirements listed above, especially the first three. User-friendly and robustness cost a fortune to achieve. Therefore, our recommendation is to trade functionality for simplicity, developing basic functions only and giving up most advanced features. Furthermore, the system will be constructed by voluntary notebook PCs. It will be unrealistic to anticipate a high performance.

\section{Performance Requirements}

- provide tolerable QoS for multimedia communications

- maintain minimum level of throughput

- $\quad$ give precedence to QoS over throughput

- $\quad$ provide class-based priority services

- provide high member-coverage for group communications

\subsection{Available Emergency Communication Systems}

There are few options for emergency communication systems.

\section{- Walkie-Talkie}

Perhaps Walkie-Talkie is the most convenient and reliable communication system for emergency. However, the popularity of Walkie-Talkie in many countries is far less than Notebook PCs..

\section{- Ham Radio}

Similar to Walkie-Talkie, Ham Radio is a very old communication technology that doesn't depend on any infrastructure to operate. It plays like a StoneAge Internet and may be useful to serve as a backup Internet when the real Internet is not available. Moreover, it is much less popular than Walkie-Talkie. In Taiwan, there are only a handful 
of Ham Radio stations due to a very long period of stringent regulation.

\section{- Emergency Mobile Communication Systems}

Various equipment vendors are offering emergency mobile communication systems [8,9]. Specially designed systems are expensive and offer only limited number of handsets. It is prohibitively expensive to establish sufficient capacity for a catastrophic disaster as big as mentioned three cases.

Most cellular operators have emergency cellular systems that use satellite links as backhaul links and can be deployed to a demanded area in a few hours. However, there are two problems. First, cellular operators may not have sufficient number of such systems for a catastrophic disaster. Secondly, volunteers do not know each other and have no time to memorize (or keep in handset) many phone numbers and may not have handset chargers in hand.

\section{- MANET based P2Pnet}

We propose to use WiFi-ready notebooks to construct a MANET based group communication system to support emergency communication and information network, called P2Pnet. In recent years, WiFi-ready notebook PC that can last several hours is becoming a very popular and universally compatible device in many areas. When stricken by a natural disaster, survivals and volunteers can use their own notebook PCs to construct a P2Pnet. Using P2P communication technologies, a P2Pnet is able to support Walkie-Talkie like communication, Push-to-Talk, VoIP, and network information systems for emergency usage. Compared with other options, no extra hardware cost is needed.

\section{MANET BASED P2Pnet}

\subsection{System Architecture}

P2Pnet is a serverless peer-to-peer communication network based on MANET to support temporary group communication and information networks. As depicted in Fig. 1 , some nodes may have satellite communication capability performing gateway functions so that all other nodes can access Internet through gateways if they are available.

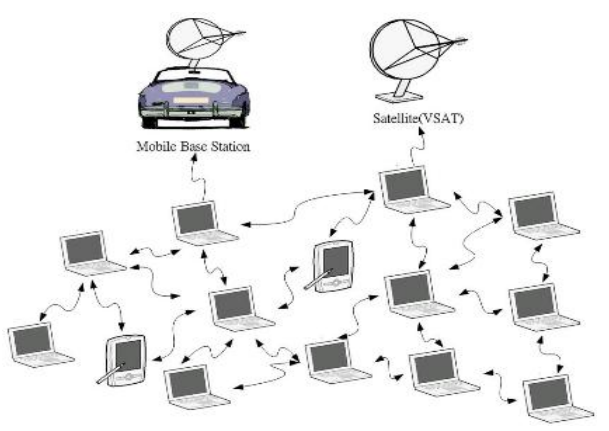

Fig. 1 Physical Architecture of P2Pnet

On top of MANET, there is a layer of peer-to-peer network service to support higher level communication services such as Walkie-Talkie, Push-to-Talk, and VoIP communications. Logical architecture is shown in Fig. 2.

The logical architecture of P2Pnet follows the traditional layered architecture with an intermediate layer between Network Layer and Transport Layer. To avoid reinventing wheel, this architecture reuses as much existing network technologies as possible.

\begin{tabular}{|c|c|c|c|}
\hline Layer & \multicolumn{3}{|c|}{ Functional Modules } \\
\hline $\begin{array}{c}\text { Application } \\
\text { Systems }\end{array}$ & $\begin{array}{l}\text { Disaster Rescue } \\
\text { Info Sys }\end{array}$ & $\begin{array}{l}\text { Mobile } \\
\text { Learning Sys. }\end{array}$ & \begin{tabular}{|l} 
Battle Field \\
Manage. Sys.
\end{tabular} \\
\hline $\begin{array}{c}\text { Application } \\
\text { Functional } \\
\text { Modules }\end{array}$ & \begin{tabular}{|l|l|} 
FTP/HTTP & PTT \\
/Telent/etc. & \\
\end{tabular} & \begin{tabular}{|l|l|} 
VolP & $\begin{array}{l}\mathrm{P} 2 \mathrm{P} \\
\text { Streaming }\end{array}$ \\
\end{tabular} & $\begin{array}{l}\text { Location-Aware } \\
\text { Applications }\end{array}$ \\
\hline Transport & \begin{tabular}{||l|l|l|} 
TCP & UDP & $\begin{array}{l}\text { Partial- } \\
\text { Reliable } \\
\text { TCP }\end{array}$ \\
\end{tabular} & \begin{tabular}{|l|l} 
Partial- & \\
Reliable & Hop-b \\
ReP \\
UDP
\end{tabular} & \begin{tabular}{l|l} 
by-hop & P2P \\
Multicasting
\end{tabular} \\
\hline $\begin{array}{l}\text { Network } \\
\text { Service }\end{array}$ & \begin{tabular}{|l} 
Uncontrolled \\
Single-Hop \\
Group \\
Communication \\
Network \\
(U1net) \\
\end{tabular} & $\begin{array}{l}\text { Uncontrolled } \\
\text { K-Hop Group } \\
\text { Communication } \\
\text { Network } \\
\text { (UKnet) }\end{array}$ & $\begin{array}{l}\text { Controlled } \\
\text { K-Hop Group } \\
\text { Communication } \\
\text { Network } \\
\text { (CKnet) }\end{array}$ \\
\hline $\begin{array}{l}\text { Network } \\
\text { Routing }\end{array}$ & Ad Hoc Network $N$ & Mesh Network & VANET \\
\hline Physical & RF Positioning Sys & stem Wi-Fi WiMA & \\
\hline
\end{tabular}

Fig. 2 Logical Architecture of P2Pnet

\section{Physical and Network Layers}

In order to use all available resources in a disastrous situation, P2Pnet will try to incorporate most available network technologies.

The most popular wireless network technologies these days are IEEE 802.11 (WiFi) and IEEE 802.16 (WiMAX). WiFi is a proven technology with very high degree of business success. Most people agree that WiFi will continue to be the dominant wireless LAN technology, while WiMAX is a newer but has not been a business success yet. Therefore, 
P2Pnet will be mainly designed on top of WiFi-Based MANET. However, WiMAX will not be excluded if it is available. Other useful technologies such as "Wireless Positioning technology" will be adopted if they are available.

At the Network Layer, many successful MANET algorithms are available ready for use. The popular AODV (Ad hoc On Demand Distance Vector) routing algorithm will be used in P2Pnet since the mobility of P2Pnet will be very high in the most applications.

Mesh network technology is another emerging WLAN technology. Although it needs an infrastructure established first before the network becomes usable, it is not that difficult to set up one. However, it may not be a practical option in a disastrous situation. Nevertheless, P2Pnet will not exclude Mesh network technology if it is available.

Another important technology is VANET. According to what we observed in Jiji Earthquake, most roads near stricken spots were either broken or jammed by disorganized vehicles that carry rescue and relief resources. In many hurricanes such as Katrina, highways near anticipated stricken cities were all jammed by the evacuation vehicles. If these vehicles were able to communicate to each other, they will be much more organized resulting in a more efficient rescue and relief operation.

Finally, the opportunistic network technology that employs store-carry-forward transmission model is useful to connect isolated P2Pnet islands into an integrated one.

\section{Network Service Layer}

Due to the loss of Internet connection, many network services may stop functioning. For instance, most VoIP (Voice-overIP) or instant messaging services require users to access centralized registration servers. Therefore, many important networking services must be reimplemented. To reduce redundancy, we designed an intermediate layer on top of the Network Layer, called Network Service Layer, to facilitate networking services at the Application Layer.

In this layer, three basic networking modes are as follows:

- U1net (Uncontrolled Single-Hop Group Communication Network) Each node can broadcast data to neighboring nodes in one-hop distance. No authorization will be enforced. This mode can support short range Walkie-Talkie like communications. Because it is the easiest to construct, it is to be deployed in the early hour of a disaster when all the organizational efforts are not in place yet.

\section{- UKnet (Uncontrolled K-Hop Group Communication Network)} Each node can broadcast data to neighboring nodes in K-hop distance. No authorization will be enforced. This mode can support long range
Walkie-Talkie like communications. This is also designed for the early hour of a disaster when all the organizational efforts are not in place yet. However, it is little more complicate than U1net so that it requires more effort to construct.

- CKnet (Controlled K-Hop Group

Communication Network)

This is a more advanced mode and can support unicast services such as VoIP. It requires more organizational effort to construct such a network mode and may not be easy to construct in the early hours of a disaster.

\subsection{Autonomous P2P Vehicular Ad-Hoc Group Communication Systems}

As mentioned in the previous section, VANET can play an important role in supporting emergency group communications. Under disastrous circumstances, the infrastructure to support a full functional VANET may not be available. Therefore, we focus on the inter-vehicle communication (IVC) technology (or peer-to-peer communication). The network environment of VANET is quite different from those of traditional wired networks and IEEE802.11 based wireless networks due to its high mobility and critical requirements of packet forwarding and routing.

Many applications have been developed due to the increasing research on the VANET related technologies. However, most researches only focus on resolving a single problem and lack of comprehensive solution to the problem. Furthermore, the environmental constraints of P2Pnet are quite different from that of a regular VANET. We thus propose an integrated solution specially designed for P2Pnet. The issues under consideration are the mobility model in a disastrous area, bandwidth resource management, disaster-specific routing and multicasting algorithms. Protocols for Network Service Layer (U1net, UKnet, and CKnet) will be tailored for VANET. Communication-specific applications such as VoIP, Push-to-Talk, and P2P Streaming will also be adapted to VANET. Other disaster-specific applications such as routing for ambulance on jammed highways will also be developed. Through these applications, we can speed up the delivery of rescue and relief resource. Although the solutions are designed specifically for P2Pnet, some technologies developed in this project are also applicable to general purpose VANET.

\subsection{Feasibility Assessment}

The availability of compatible notebook PCs and power supplies are the two most critical feasibility concerns.

\section{Availability of Notebook PC}

In many countries, highly compatible notebook PCs equipped with 802.11 WLAN capability running TCP/IP network protocol is getting more and more popular. Starting from 2008, inexpensive notebook PC has been very successful and 
has no sign to slow down. Proliferation of computer literacy to the low income world in the near future has been in our vision. We can imagine that constructing a P2Pnet for emergency communication is completely feasible in many areas of the world. It would be foolish to ignore such a convenient communication tools.

\section{Availability of Power Supplies}

Electricity will be most likely knocked out even in a small disaster such as a snow storm. Power generator has long been a typical equipment in most contingency plans. In some areas such as Taiwan, portable generators are very popular because of a large population of flea-market-style evening markets.

On the other hand, the battery life has been extended from 2 hours to 8 hours. As mini-notebook PC, that consumes less power than regular notebook PCs, gets its momentum recently, we can anticipate that the chance of having many long-life notebook PCs in many areas is very high. Compared with other heavy machinery, the fuel consumed by P2Pnet is only a small fraction of total fuel consumption.

\subsection{System Developments and Deployment}

P2Pnet has been developed in two phases. The first phase is to develop a simple MANET. Only simplified information service is supported. In the second phase, a more advanced system will be constructed. It will be able to support broadcast-based and unicast-based multimedia communications such as Walkie-Talkie and VoIP conference.

When a disaster strikes suddenly, there is not much time allowed to deploy a full-functional P2Pnet. The simplest network function, U1net can be deployed first to support Walkie-Talkie like communications in the early hour of rescue operation. If time is allowed, UKnet and CKnet can be deployed in turn to establish a more advanced network as well as full functional VoIP service. The design details are as follows.

\section{- 1Knet, UKnet, and CKnet}

1 Knet and UKnet can be quickly deployed using the 255.255.255.255 broadcast address when unique IP addresses are not available. To support CKnet, unique IP addresses must be available. Each node proceeds to discover its immediate neighbors first and then exchange routing information with its neighbors to build up its own routing table.

\section{- Neighbor Discovery}

Using the broadcast address 255.255.255.255 and UDP socket, each node can discover its immediate neighbors by sending a 'hello' message containing its own IP address and a response port number to all its neighbors. All the neighbors who hear the message can send back their IP addresses through the designated port, and then try to coordinate a common port number for future communications.

\section{- Internet Access}

There are several options to provide Internet access to the P2Pnet for emergency: satellite communications (VSAT or Iridium) or mobile data services (such as $3 \mathrm{G}$ ) if there are available. A mobile gateway is designed to offer shared Internet access for all the nodes in a P2Pnet.

\section{EXPERIMENTS}

We designed a Rescue Information System for Earthquake Disaster (RISED) [2, 3] and a mobile learning platform over P2Pnet, NCCU-MLP[1]. We also conducted a research based on NCCU-MLP to study the effectiveness and the behavior of a group of English learning students as well as to test our P2Pnet [1]. (Note that it is unrealistic to test our design in a real catastrophic earthquake.)

\subsection{Rescue Information System for Earthquake Disasters}

RISED is designed to support a more efficient rescue and relief operation for catastrophic earthquake disasters. A disaster area may lose its connection to the external world for numerous reasons. As a consequence, existing rescue information networks may stop functioning resulting in efficiency degradation of rescue operations. The objective of the RISED is to provide the most up-to-date rescue-related information such as disaster locations, possible damages to both lives and constructions, available rescue and relief resources, the shortest way to the disaster spots, etc. The RISED will help to better manage the rescue and relief resources such that these resources can be allocated to the neediest areas in time. The decision support system is deployed in a two-tier architecture. The first-tier is the central RISED and the second-tier is the local RISED. The central RISED, deployed in the central rescue command center, is responsible for nationwide disaster assessment and resource management. A local RISED, deployed in a local rescue command center, collects local seismic and rescue related data and uploads to the central RISED periodically at ordinary days. It can operate independently to help rescue and relief operation when the responsible region is stricken by an earthquake and lose its connection to the external world. If an emergency P2Pnet has accesses to the Internet, the central and local RISEDs can exchange information for a more accurate disaster assessment as well as a better management on the rescue operation. If, unfortunately, the emergency P2Pnet has no access to the Internet, local RISEDs can operate independently. The ER diagram of RISED is shown in Fig. 3. 


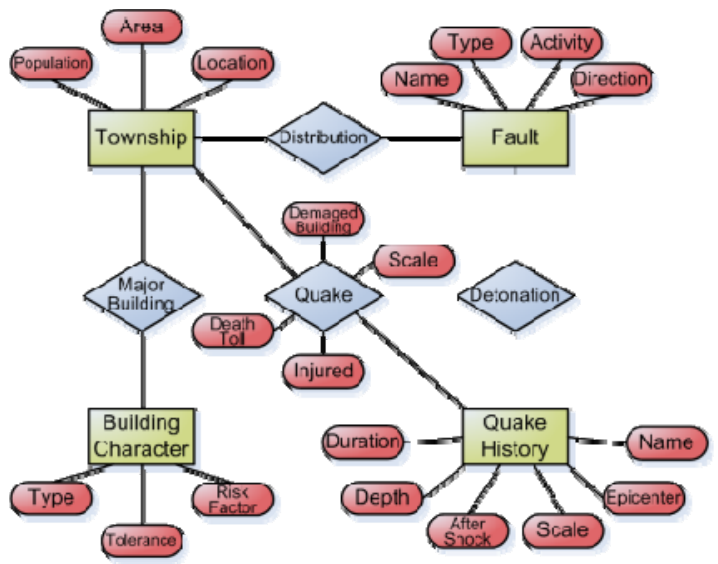

Fig. 3 ER Diagram of RISED

RISED provides seismic related information to assist disaster assessment. Useful information includes seismic fault locations, population distributions, resource distributions, building characteristics, road information and quake history. Together with collected real-time disaster statistics, the information system can support a more accurate disaster assessment for a better distribution of rescue and relief resources.

\subsection{A Study of Mobile Learning}

This research studies the effectiveness and the behavior of a group of English learning students over a mobile learning platform (NCCU-MLP) developed in National Chengchi University (NCCU). The goal of NCCU-MLP is to improve students' English ability as well as to update teachers' teaching using the latest technology. It offers a multimedia based English learning environment as well as Push-to-Talk (PTT) and whiteboard capability for group communications. The technology aspects of the experiment results are summarized in this section.

- The transmission quality of wireless radio signal is highly dependent on the weather conditions, especially on rainy days. As a consequence, the stability of mobile network connection is lower than fixed networks. The design of mobile learning environment must take this into consideration.

- The software system in a mobile computing environment is much more complicated than that on fixed networks. Thus, it needs more effort to make the software system robust.

- Compared with voice communication tool, like PTT, whiteboard is even less ideal for group communication. First, people are used to talk than to write. Secondly, voice communication is much more convenient than that of hand-writing.

- Users prefer full-duplex conversation mode to halfduplex mode. However, it remains a great technical challenge to offer group voice communication in full-duplex conversational mode under limited bandwidth.

\section{CONCLUDING REMARKS}

One of the most important lessons we learned from numerous disasters is that mobile communication system is vulnerable and the loss of communication system may have a catastrophic consequence. This paper analyzes the causes that paralyzed the entire communication systems in Jiji Earthquake and propose a P2Pnet that uses notebook PCs to construct a MANET based emergency communication and information system. Brief system requirements and system design are presented. The architecture of P2Pnet and the Rescue Information System for Earthquake Disasters are presented. Finally, a P2Pnet prototype was tested in an English mobile learning class.

\section{REFERENCES}

1. Pei-Chun Che, Han-Yi Lin, Hung-Chin Jang, Yao-Nan Lien and Tzu-Chieh Tsai, 2004, "A Study of English Mobile Learning Applications in National Chengchi University", Submitted to Journal of Distant Learning Technology.

2. Hung-Chin Jang and Tzu-Chieh Tsai, "Mobile Information Management System for Disastrous Earthquake Emergency," FET Labs Journal, Vol. 3, 2001, pp. 64-68.

3. Yao-Nan Lien, Tsu-Chieh Tsai and Hung-Chin Jang, "A Mobile Information Management System for Disastrous Earthquake Emergency", Proc. of the 6th Workshop on Mobile Computing, March 2000, pp. 151-154.

4. Yao-Nan Lien, Hung-Chin Jang and Tsu-Chieh Tsai, "A MANET Based Emergency Communication and Information System for Catastrophic Natural Disasters ", to appear in the IEEE Workshop on Specialized Ad Hoc Networks and Systems, June 26, 2009.

5. Jiji Earthquake, http://jiji.ncree.gov.tw/, retrieved Dec. 19, 2008. Hurricane Katrina, http://en.wikipedia.org/wiki/Hurricane_Katrina, retrieved Dec. 19, 2008.

6. Hurricane Katrina, http://en.wikipedia.org/wiki/Hurricane Katrina, retrieved Dec. 19, 2008.

7. SiChuan Earthquake, http://en.wikipedia.org/wiki/2008_Sichuan_earthquake, retrieved Dec. 19, 2008.

8. http://www.ideal-ist.net/Countries/DE/PS-DE-486, retrieved Dec. 19, 2008.

9. http://www.gothamgazette.com/article/20040524/19/9 90, retrieved Dec. 19, 2008. 